\title{
REFLEXIONES SOBRE LA VIOLENCIA DE GÉNERO DURANTE EL CONFINAMIENTO CAUSADO POR COVID-19
}

\author{
Estrella Fátima Rueda Aguilar \\ Universidad de Sevilla \\ España
}

\begin{abstract}
RESUMEN
Durante el periodo de confinamiento, de marzo a junio de 2020, las mujeres asesinadas víctimas de la violencia machista fueron ocho, 11 menos que en el mismo periodo del año anterior. Además, el número de denuncias disminuyó $10.25 \%$ en el primer cuatrimestre y $14,62 \%$ en el segundo cuatrimestre de 2020 . No obstante, se confirmó un incremento de $41.1 \%$ en llamadas al 016 desde el 14 de marzo al 31 de mayo. Lo que en principio podía indicar una disminución de la violencia de género, en realidad ponía de manifiesto dificultades de las víctimas para pedir ayuda durante el confinamiento, evidenciado sobre todo por el incremento del uso de medios silenciosos. El presente estudio hizo una revisión bibliográfica para reflexionar acerca de cómo ha influido el Covid-19 en la violencia de género, en su vulnerabilidad y cuáles han sido las consecuencias. La literatura actual revisada indicó que las condiciones generadas por la pandemia por Covid-19 ha impactado de modo negativo en la violencia de género porque las mujeres víctimas se han encontrado aisladas sin ningún tipo de apoyo familiar o social. El aumento de la vulnerabilidad también se manifiesta mediante la sintomatología depresiva. Por último, se aportan datos importantes a tener en cuenta a la hora de implementar intervenciones.
\end{abstract}

Palabras Clave:

Covid-19, violencia de género, pandemia, confinamiento, riesgo de violencia

\section{REFLECTIONS ON GENDER VIOLENCE DURING CONFINEMENT CAUSED BY COVID-19}

\begin{abstract}
During the period of lockdown, from March to June 2020, there were eight murdered women victims of gender-based violence, eleven less than in the same period of the last year. In addition, the number of complaints decreased by $10.25 \%$ in the first quarter and by $14.62 \%$ in the second quarter of 2020 . However, an increase of $41.1 \%$ in calls to 016 was confirmed from March 14 to May 31. At first it could indicate a decrease in gender-based violence, revealed difficulties for victims to ask for help during lockdown, evidenced mainly by the increase in the use of silent means. This study carried out a bibliographic review to reflect on how Covid-19 has influenced gender-based violence, its vulnerability and what the consequences have been. The literature reviewed indicated that the conditions generated by the Covid-19 pandemic have had a negative impact on gender-based violence, since female victims have been isolated without any type of family or social support. Increased vulnerability has also manifested itself through depressive and affective symptoms. Finally, important data is provided to consider when implementing interventions.
\end{abstract}

Keywords:

Covid-19, gender-based violence, pandemic, lockdown, violence risk

Bitácora del ARtículo:

| Recibido: 02 de Abril de 2021 | Aceptado: 28 de Junio de 2021 | Publicado en línea: Julio-Diciembre de 2021 | 
Revisión de la literatura | Reflexiones sobre la violencia de género durante el confinamiento... Rueda Aguilar

Autoría y Derechos de Propiedad Intelectual

\title{
REFLEXIONES SOBRE LA VIOLENCIA DE GÉNERO DURANTE EL CONFINAMIENTO CAUSADO POR COVID-19
}

\author{
Estrella Fátima Rueda Aguilar \\ Universidad de Sevilla \\ España
}

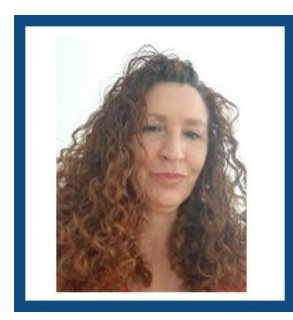

Estrella Fátima Rueda Aguilar

Universidad de Sevilla

Correo: erueda@us.es

\begin{abstract}
Doctora en Ciencias Sociales por la Universidad Pablo de Olavide. Especialista en Psicología Clínica. Experta en investigaciones feministas y en perspectiva feminista del pensamiento científico. Experta en formación de formadores. planificación, diseño, evaluación de proyectos y programas. Profesora en el Departamento de Psicología Social de la Universidad de Sevilla (España). Profesora-Tutora Universidad Nacional de Educación a Distancia. Profesora consultora en Ciclo Formativo de Integración Social en la Universitat Oberta de Catalunya. Amplia experiencia en el ámbito del a intervención social.
\end{abstract}

\section{CONTRIBUCIÓN DE los Autores}

Responsable de la idea y coordinación del proyecto, búsqueda bibliográfica en la base de datos, revisión, redacción y supervisión del manuscrito.

\section{AGRADECIMIENTOS}

Se agaradece a todas las personas involucradas en la realización de esta investigación.

\section{Datos de FiLiación de los Autores}

Departamento de Psicología Social de la Universidad de Sevilla

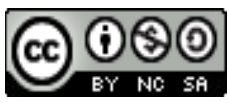

Copyright: (C) 2021 Rueda-Aguilar, E. F,

Este es un artículo de acceso abierto distribuido bajo los términos de la licencia Creative Commons Reconocimiento-NoComercial 4.0 Internacional, por lo que su contenido gráfico y escrito se puede compartir, copiar y redistribuir total o parcialmente sin necesidad de permiso expreso de sus autoras con la única condición de que no se puede usar con fines directamente comerciales y los términos legales de cualquier trabajo derivado deben ser los mismos que se expresan en la presente declaración. La única condición es que se cite la fuente con referencia a la Revista Digital Internacional de Psicología y Ciencia Social y a sus autoras. 


\section{TABLA DE CONTENIDO}

INTRODUCCIÓN

MÉTODO

RESULTADOS

Consecuencias de la pandemia en la violencia de género, ¿Tiene la pandemia por Covid-19 un impacto negativo en la violencia de género?, 187

Confinamiento e influencias de la violencia de género, 189

Vulnerabilidad, Covid-19 y violencia de género, 190

\section{Discusión}

CONCLUSIÓN

REFERENCIAS 


\section{INTRODUCCIÓN}

$\mathbf{L}$ a violencia contra la mujer es una de las violaciones de derechos humanos más presente en el mundo, causada por la desigualdad de género, la discriminación y las dañinas normas culturales y sociales. Cada vez se reconoce más como un tema de salud pública que afecta de modo negativo a la salud de las mujeres, y su grave impacto la ha convertido en uno de los temas más importantes de nuestro tiempo.

En la literatura publicada referente al tema es muy difícil encontrar un consenso en la definición de "violencia de género", porque la naturaleza de la violencia que sufren las mujeres en sus relaciones íntimas es muy compleja (Heise, Pitanguy y Germain, 1994), coexistiendo distintos términos que con diferentes niveles de generalidad intentan abarcar este fenómeno. Osborne (2009) clasifica las distintas conceptualizaciones:

1. Violencia doméstica. Reduce la violencia de género al contexto doméstico, e incluye a todas las personas que habitan en ese ámbito, independientemente de su género y tipo de relación. Este término invisibiliza y niega la violencia de género que sufren las mujeres que no conviven con las parejas agresoras.

2. Violencia familiar. Abarca a mujeres y hombres que tienen algún tipo de relación familiar más allá de la relación de pareja.

3. Violencia contra las mujeres. Define quién es la víctima principal de esta violencia. Este término invisibiliza la naturaleza de esta violencia (sexismo, machismo, patriarcado), e incluye cualquier violencia hacia cualquier mujer, incluidas las que no son por razón de género.

4. Violencia de género. Es una extendida violación de los derechos humanos. La Ley Orgánica 1/2004 de 28 de diciembre, de Medidas de Protección Integral contra la Violencia de Género en España la define en su artículo 1.1.:

"[...] como manifestación de la discriminación, la situación de desigualdad y las relaciones de poder de los hombres sobre las mujeres, se ejerce sobre éstas por parte de quienes sean o hayan sido sus cónyuges o de quienes estén o hayan estado ligados a ellas por relaciones similares de afectividad, aun sin convivencia.
[...] comprende todo acto de violencia física y psicológica, incluidas las agresiones a la libertad sexual, las amenazas, las coacciones o la privación arbitraria de libertad" (p. 42168).

Una de las complejidades que presenta esta ley es que supedita la violencia de género a la ocurrencia de ésta dentro de una relación de afectividad, dejando fuera otros tipos de violencia derivados del machismo y el sexismo, como la trata de mujeres y niñas o la prostitución.

Según Corsi (1994) la violencia de género se refiere a todas las formas mediante las cuales se intenta perpetuar el sistema de jerarquías impuesto por la cultura patriarcal, por lo que se trataría de una violencia estructural que se dirige hacia las mujeres con el objetivo de mantener o incrementar su subordinación al género masculino hegemónico. De esta manera, la violencia de género adoptaría formas muy variadas, tanto en el ámbito público como en el privado, evidenciándose sus distintas formas de discriminación hacia la mujer en diferentes niveles (político, institucional, laboral), y también en el acoso sexual, la violación, el tráfico de mujeres para prostitución, la utilización del cuerpo femenino como objeto de consumo, la segregación basada en ideas religiosas, además de en todas las formas de maltrato físico, psicológico, social y sexual que sufren las mujeres en cualquier contexto, y que ocasionan una escala de daños que pueden culminar en la muerte.

Siguiendo a Nogueiras (2005), la violencia de género se caracteriza por:

- Ser ejercida por el hombre con el que se mantiene o se ha mantenido un vínculo afectivo-sexual.

- No ser un hecho aislado; es un proceso a medio-largo plazo que debilita gradualmente a las mujeres que la sufren.

- Suele producirse en espacios o ámbitos de intimidad, por lo que muchas veces son invisibles para las personas que rodean a la pareja.

- Tiende a ocultarse, tanto por quien agrede como por la persona que sufre la violencia.

- El hombre que ejerce la violencia suele tener una adecuada imagen pública; es valorado socialmente.

- Las mujeres que la sufren sienten vergüenza y culpabilidad, lo que las frena a contarlo.

- La socialización discriminatoria de género potencia en las mujeres valores y actitudes como la comprensión y la empatía, que después se vuelven en su contra llegando incluso a justificar la violencia de género. 
- Su reforzamiento a través de los mitos.

- Su breve reconocimiento como problema social de extrema gravedad.

- La dificultad para reconocer que detrás de la violencia se encuentran hombres socializados en un tipo de masculinidad que fomenta estas agresiones.

En pleno siglo XXI la violencia de género aún es un problema de salud pública grave a nivel mundial. Las circunstancias de crisis sanitaria, derivadas de la pandemia causada por el Covid-19 han ocasionado que hoy este problema se convierta en uno de los principales focos de atención y de emergencia debido a la constatación del aumento de la violencia.

Desde que en España se contabilizan las víctimas mortales por violencia de género (2003), 1,081 mujeres han fallecido a manos de sus agresores; de éstas, 45 han perdido la vida en 2020. En la tabla 1 se muestran cifras de las víctimas mortales por violencia de género en los últimos cinco años (2016-2020).

Considerando que en España se vivió un confinamiento desde el 15 de marzo hasta el 21 de junio de 2020, el análisis cuantitativo de mujeres asesinadas en este periodo indica que hay un descenso de mujeres víctimas mortales de violencia de género (tabla 1). En los últimos cinco años (2016-2020) y en el periodo definido por el confinamiento debido al Covid-19 (de marzo a junio) se informó de 12 mujeres asesinadas por violencia de género en 2016, y en años posteriores se produjo un aumento, contabilizándose 15 mujeres en 2017, 14 en 2018 y 19 en 2019; aunque en 2020 se observa una disminución, registrándose ocho mujeres asesinadas durante el confinamiento, 11 menos que el año anterior (Ministerio de Sanidad, Servicios Sociales e Igualdad [MSSSI], 2020).

Por otro lado, destaca un aumento durante el confinamiento en la utilización del servicio telefónico de información, asesoramiento jurídico y atención psicosocial inmediata a todas las formas de violencia contra las mujeres, a través del número telefónico de marcación abreviada 016. Desde la segunda quincena de marzo comenzó a observarse un incremento significativo en comparación con el mismo mes del año anterior, consolidándose una tendencia creciente reflejada en un repunte del número de Ilamadas. En general, las Ilamadas a las líneas de atención han aumentado en todo el mundo, y en España, según la Delegación de Gobierno contra la Violencia de Género (Ministerio de Igualdad) (2020), se confirma un incremento de $41.1 \%$ desde el 14 de marzo al 31 de mayo. Además, las Ilamadas "on line" crecieron $450 \%$ en relación con el mismo periodo de 2019, y se recibieron 2,038 Ilamadas desde el 21 de marzo a 31 de mayo en el nuevo servicio de apoyo emocional y psicológico a las víctimas a través de WhatsApp.

Tabla 1.

Mujeres víctimas mortales de violencia de género entre 2016 y febrero 2021 en España.

\begin{tabular}{lllllll} 
& 2016 & 2017 & 2018 & 2019 & 2020 & 2021 \\
\hline Enero & 7 & 5 & 2 & 8 & 7 & 1 \\
Febrero & 4 & 10 & 2 & 3 & 6 & 2 \\
\hline Marzo & 2 & 3 & 3 & 4 & 4 & - \\
Abril & 4 & 4 & 4 & 5 & 1 & - \\
\hline Mayo & 4 & 6 & 1 & 3 & 2 & - \\
\hline Junio & 2 & 3 & 6 & 7 & 1 & - \\
\hline Julio & 6 & 2 & 8 & 10 & 4 & - \\
Agosto & 4 & 4 & 7 & 3 & 8 & - \\
\hline Septiembre & 2 & 2 & 10 & 4 & 4 & - \\
Octubre & 4 & 5 & 4 & 4 & 1 & - \\
\hline Noviembre & 5 & 3 & 2 & 3 & 3 & - \\
Diciembre & 5 & 3 & 2 & 1 & 4 & - \\
\hline Total & 49 & 50 & 51 & 55 & 45 & 3 \\
Fuente: MSSSI (2020). & & & & \\
\hline
\end{tabular}

Aunque en principio los datos expuestos podrían indicar una disminución de la violencia de género en la mujer, en realidad ponen de manifiesto dificultades de las víctimas para pedir ayuda durante el confinamiento, evidenciadas fundamentalmente por el incremento de la utilización de medios silenciosos, como las Ilamadas al 016, Ilamadas online o el servicio de apoyo emocional y psicológico a través de WhatsApp.

Las denuncias por violencia de género durante el periodo de confinamiento disminuyeron según informes de los Juzgados de Violencia sobre la Mujer, pese al normal funcionamiento de éstos. Es evidente la dificultad añadida que el confinamiento aporta al interponer una denuncia por las limitaciones de movilidad y autonomía para salir, además de la dificultad para visibilizar los problemas de violencia que se dan dentro de los domicilios. En este sentido es necesario destacar que, de las 38 víctimas por violencia de género en 2020, sólo ocho habían interpuesto denuncia. Según el Consejo General de Poder Judicial (2020), los juzgados recibieron 36,185 denuncias por violencia de género en el primer trimestre, y 34,576 en el segundo trimestre, lo que supone una variación de $-10.25 \%$ y $-14.62 \%$ respectivamente, cuando se comparó con el mismo periodo del 
año anterior. De nuevo estos datos no implicaban que la violencia de género había disminuido, sino que las condiciones circunstanciales del confinamiento limitaban de modo fundamental la libertad de movimiento de las víctimas, y por tanto para poder cursar una denuncia.

La actual pandemia Covid-19 ha dado lugar a un aumento de los niveles de violencia de género en todo el mundo (Taub, 2020). Este crecimiento es tan crítico que ha sido descrito como una doble pandemia (Bettinger-López y Bro, 2020) y una nueva crisis (Taub, 2020). Sin embargo, hay que tener en cuenta que, pese a que es evidente el crecimiento de riesgos de la violencia de género en esta situación, es necesario indicar que a veces no son percibidos por los procedimientos habituales establecidos para su detección, y que no se reflejan de manera directa en los datos estadísticos publicados.

Investigaciones previas han proporcionado evidencias que indican un aumento de los niveles de violencia durante o después de desastres naturales y eventos catastróficos (Zahran, Shelley, Peek y Brody, 2009). Más concretamente, han demostrado de modo exhaustivo que la violencia de género aumentó después los desastres naturales (Gearhart et al., 2018), como terremotos (Chan y Zhang, 2011), inundaciones (Gearhart et al., 2018) y huracanes (Anastario, Shehab y Lawry, 2009) porque los agresores aprovechan las circunstancias en las que se restringe la circulación para incrementar el uso de la violencia contra las mujeres (Sakurai et al., 2017). Distintos estudios han mostrado que después de diferentes desastres (el terremoto de Haití, los incendios en Australia y el tsunami en Japón) se observó un aumento de la violencia de género en dos niveles diferentes; por un lado, un aumento del nivel de violencia en las relaciones en que ya sucedía, y por otro lado la aparición de violencia de género en relaciones en las que antes no existía, y todo ello sin aumentar el número de denuncias (Parkinson, 2019). Campbell (2020) indica que el aumento de violencia durante y después de la pandemia de Covid-19 puede incluso exceder a los grandes incrementos observados en los informes que siguen desastres y otros sucesos catastróficos.

El confinamiento vivido como medida para contener el avance del Covid-19 no ha sido una medida de seguridad y protección para las víctimas de violencia de género, que se han visto obligadas a convivir las 24 horas con sus agresores, aumentando con esta situación la vulnerabilidad de este colectivo al tener que vivir con un constante doble temor, a su agresor y al virus, y observándose sobre todo un incremento de la tensión y el estrés, y un aumento del aislamiento caracterizado por el alejamiento de sus apoyos sociales y de los recursos dis- ponibles (Gallo y Mañas, 2020). Así en el apartado I del Real Decreto-Ley 12/2020, de 31 de marzo, de medidas urgentes en materia de protección y asistencia a las víctimas de violencia de género de España se indica:

\begin{abstract}
"Las mujeres víctimas de violencia de género son un colectivo especialmente vulnerable en situaciones de aislamiento domiciliario, por verse forzadas a convivir con su agresor, lo que las sitúa en una situación de mayor riesgo, como se ha venido demostrando con motivo de situaciones parcialmente análogas, como los periodos vacacionales sin situación de permanencia en domicilios, periodos en los que se disparan los casos de violencia de género y de violencia doméstica" (p. 27973).
\end{abstract}

En el presente estudio se realiza una revisión bibliográfica para reflexionar acerca de cómo ha afectado el Covid-19 y la situación de confinamiento vivida en mujeres víctimas de violencia de género, y entender cómo Covid-19 puede llegar a exacerbar los factores que conducen a una escalada de violencia. Teniendo en cuenta este planteamiento se propone dar respuesta a las siguientes cuestiones: 1) ¿ha tenido la pandemia por Covid-19 un impacto negativo en la violencia de género?; 2) ¿se identifican cuáles han sido las influencias específicas del confinamiento en la violencia de género?, y 3) ¿aumentó la vulnerabilidad de las mujeres sometidas a violencia de género durante la pandemia por Covid-19?

\section{MÉTodo}

Para desarrollar este trabajo se utilizó el enfoque cualitativo descriptivo por medio del método bibliográfico para indagar, recolectar, organizar, analizar e interpretar informaciones (Alfonso, 1995) acerca de la violencia del género durante el confinamiento. El propósito fue la construcción de conocimientos y la sistematización objetiva y metódica de los estudios publicados con el objetivo de concretar el estado de arte de la cuestión (Sánchez-Meca, 2010). Para la localización de documentos se utilizaron varias fuentes documentales. Se hizo una búsqueda sistemática de publicaciones académicas con el buscador Google Scholar y en las bases de datos de Psicodoc y Psyclnfo, utilizando los siguientes descriptores en español y en inglés para la búsqueda: "Violencia de Género/Gender Violence", "Confinamiento/Lockdown" y "Covid-19".

Los criterios de inclusión utilizados para la selección de los artículos fueron: 
1. Publicados en las bases de datos Psicodoc y Psyclnfo durante los ocho meses posteriores a la declaración del estado de alarma por la pandemia de Covid-19 en España, es decir del 15 de marzo al 15 de noviembre de 2020.

2. Centrado en algún aspecto psicosocial (psicológico, emocional, relacional y/o social) de la violencia de género.

3. Idioma: inglés o español.

A partir del número inicial de trabajos obtenidos $(\mathrm{N}=52)$, se hizo un primer cribado para seleccionar sólo las publicaciones que cumplían los criterios de inclusión establecidos, resultando 24 artículos que fueron almacenados en el gestor bibliográfico Mendeley. Se utilizó la ficha bibliográfica como instrumento de registro. La extracción de la información fue hecha por dos colaboradoras independientes.

La figura 1 muestra el diagrama de búsqueda. Se han tenido en cuenta la guía y algunas directrices propuestas por la declaración Prisma, en aras de hacer un análisis que garantice sistematicidad y estandarización durante el procedimiento (Urrutia y Bonfill, 2010).

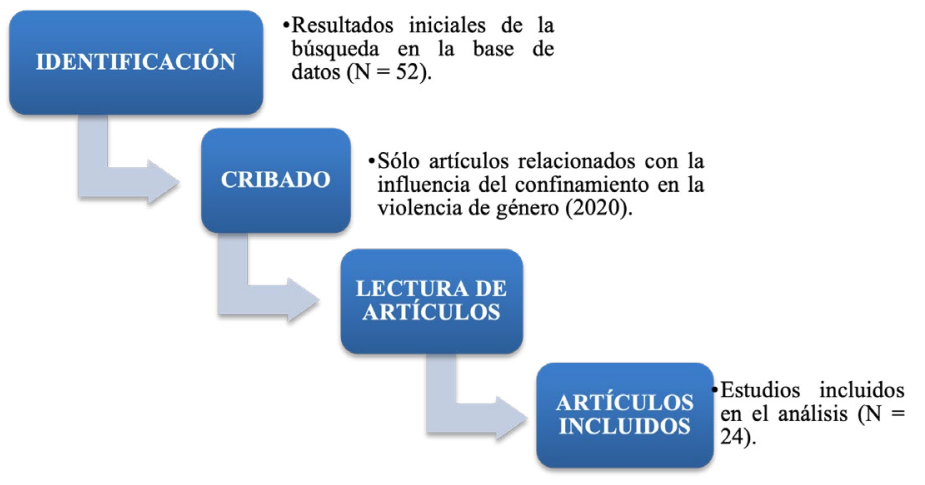

Figura 1.

Diagrama de búsqueda.

Fuente: Elaboración propia.

\section{Resultados}

Debido a la aparición del Covid-19 se dispuso a nivel mundial un confinamiento social obligatorio, que implicaba permanecer en aislamiento durante un periodo definido para tratar de evitar o limitar que se extendiera el contagio de la enfermedad. Esta circunstancia evidenció tremendas desigualdades económicas, sociales y políticas (López-Hernández y Rubio-Amores, 2020) que incrementaban la exposición a riesgos, y por tanto la vulnerabilidad de las mujeres a sufrir violencia. Un informe del Fondo de Población de las Naciones Unidas (UNFPA, 2020) indicó:

\begin{abstract}
"Las pandemias exacerban las desigualdades de género y las vulnerabilidades existentes, lo cual aumenta los riesgos de abuso. En épocas de crisis, como sucede durante un brote, las mujeres y las niñas pueden presentar un riesgo más elevado, por ejemplo, de padecer violencia infligida por la pareja y otras formas de violencia intrafamiliar como resultado de las tensiones crecientes en el hogar. También enfrentan mayores riesgos de otras formas de violencia de género, incluidas la explotación y el abuso sexuales en estas situaciones" (p. 6).
\end{abstract}

\section{Consecuencias de la pandemia en la violencia de género, ¿Tiene la pandemia por Covid-19 un impacto negativo en la violencia de género?}

Todavía hay mucho que aprender acerca de esta pandemia y el impacto que tendrá en nuestra sociedad. No obstante, ya se sabe que la pandemia ha sido un trauma debido a su naturaleza, por lo que si una persona no puede escapar del abuso durante este periodo de aislamiento social, el riesgo de abuso aumenta (Bagwell-Gray y Bartholmey, 2020). La situación generada por la pandemia de Covid-19 aumentó las desigualdades sociales, impactando de modo grave los grupos más vulnerables y a las personas con escasas redes de contención, como ocurre con las mujeres víctimas de violencia de género, quienes han quedado atrapadas en sus hogares junto a sus maltratadores (Montenegro y Montenegro, 2020). Alteraciones drásticas de la normalidad comúnmente asociadas con pandemias exacerbaron las ocurrencias de la violencia de género, sobre todo por el estrés y la inestabilidad del empleo, la pobreza y el aislamiento social (Van Gelder et al., 2020), este último utilizado como táctica por los abusadores para distanciar a las víctimas de sus redes de apoyo (Coohey, 2007).

A pesar de los limitados precedentes, algunas investigaciones (Campbell, 2020) indican que los efectos de las pandemias son sobre todo rápidos, con un aumento de la presión, cambios repentinos en el trabajo diario, cierre de las escuelas y los recursos comunitarios, y en general una reducción de los recursos disponibles. Montenegro y Montenegro (2020) manifestaron que las situaciones de vulnerabilidad de las poblaciones con menos recursos se agravaron con la pandemia derivada por el Covid-19 sobre todo por la pérdida de empleos, la precarización laboral, la alteración de las rutinas diarias, el mayor número de episodios de ansiedad y depresión, el crecimiento de adicciones, el aumento de casos de violencia doméstica e intrafamiliar en mujeres, niñas, niños y adolescentes, y 
en casos más graves femicidios, todo esto pese a que algunos autores cuestionaran la evidencia científica de que el confinamiento causara un aumento de la violencia de género (Reingle-Gonzalez et al., 2020).

Leslie y Wilson (2020) incidieron en que el aumento del aislamiento familiar, del desempleo y del estrés económico son los grandes potenciadores de la violencia de género. Los estudios de Zhang (2020) en China sugirieron un aumento en general de la violencia durante la pandemia de Covid-19 como consecuencia del agravamiento de los conflictos familiares, la angustia económica, la tensión causada por la pandemia entre los miembros de la familia, y un inadecuado apoyo a las víctimas durante la pandemia.

Por su parte, Marques et al. (2020) indican que los factores que pueden agravar la violencia de género se relacionan con las restricciones de movimiento y el encierro, los problemas y la inseguridad relacionados con un empeoramiento en la economía, el aumento de estrés del agresor, el temor a contagiarse de Covid-19 y las dudas respecto al futuro, la falta de contacto social y el consumo de sustancias psicotrópicas.

En resumen, la precariedad económica ocasionada por la crisis y las medidas de austeridad impuestas influyeron de modo grave en la violencia contra las mujeres. Es importante tener en cuenta el aumento del desempleo, la temporalidad y la inestabilidad laboral, la dependencia económica o la sobrecarga de tareas reproductivas como elementos facilitadores (Ruiz-Pérez y Pastor-Moreno, 2020). Algunas investigaciones previas referentes al desempleo, la pérdida de empleo y la inseguridad financiera evidenciaron que son factores estresantes que puede conducir a un aumento de la violencia familiar y de género (Bowlus y Seitz, 2006).

Los académicos indican que los bajos ingresos están relacionados con el aumento de la violencia en los hogares y plantean:

1. Aunque en una familia no existieran antecedentes de violencia de género, la angustia económica durante una pandemia por las tensiones financieras y la falta de apoyo social podía alimentar la violencia. Esto es consistente con la literatura que indica que niveles altos de estrés en las parejas aumentan la tasa de violencia 3.5 veces (Peprah y Koomson, 2017).

2. La violencia doméstica, y de género, podía estar impulsada por un cambio de responsabilidades. Investigaciones recientes sugieren que el confinamiento ha dado lugar a un crecimiento en la carga que soportan las mujeres en las ta- reas dedicadas a los cuidados del hogar o de personas a su cargo (Gallo y Mañas, 2020).

3. La violencia de género podría surgir como consecuencia de un aumento de interacciones, debido a que se pasa más tiempo junto al maltratador en casa. Los confinamientos, las órdenes de quedarse en casa y las pautas de distanciamiento social influyeron en que los maltratadores pasen más tiempo en casa (Jarnecke y Flanagan, 2020; Borges, 2020).

En esta misma línea, Sharma y Borah (2020) pusieron de manifiesto claros vínculos entre Covid-19 y la violencia doméstica, que a su vez impactó en la crisis económica y social. Los despidos, la pérdida de ingresos y las estancias domésticas prolongadas debido a las órdenes de quedarse en casa aumentaron la incidencia de violencia doméstica.

Por otro lado, es bien conocida la relación entre la violencia de género y los problemas de salud en las víctimas (Sabri et al., 2020), no sólo derivadas de lesiones graves a consecuencia de la violencia, sino también las dolencias crónicas con consecuencias a largo plazo en la salud física y mental (Gallo y Mañas, 2020). La reclusión con el agresor a traduce en multiples efectos en la salud de la mujeres, en su binestar, en su salud física y sexual, en su salud mental y en la salud de sus hijos e hijas (Izquierdo, 2020).

Algunos autores indican las dificultades de las mujeres víctimas de violencia de género para el acceso a recursos de apoyo durante el periodo de confinamiento. Con la crisis sanitaria derivada del Covid-19, la mayoría de los servicios se dirigieron a los enfermos de coronavirus, relegando a un segundo plano a los afectados por violencia intrafamiliar o de género y otras problemáticas sociales (Marques et al., 2020). Tradicionalmente los servicios de salud han sido los principales medios para establecer contacto con las mujeres víctimas de violencia de género. En la situación de emergencia, todas las barreras que tradicionalmente han dificultado la detección eficaz de la violencia de género, como la falta de tiempo o la poca formación para abordar esta problemática, se agudizó de nuevo debido a la sobrecarga de trabajo de los profesionales (Peterman et al., 2020). Por tanto, es posible que la situación de crisis afectara de manera negativa la capacidad de los profesionales para detectar y atender de modo adecuado los casos de violencia de género porque, además, se constató una reducción de recursos humanos y de recursos o servicios a los que las mujeres podían ser derivadas (Otero-García et al., 2018). Además, fue evidente el impacto en el acceso a la justicia y en el siste- 
ma de seguimiento institucional para mujeres víctimas de violencia de género (Carril, 2020).

En la tabla 2 se muestran las principales consecuencias de la pandemia por el Covid-19 analizadas.

\section{Confinamiento e influencias de la violencia de género}

La declaración de confinamiento generó un impacto negativo en la seguridad de la mujeres porque potencian los factores de riesgo de la violencia de género a nivel individual, familiar y social al aumentar el aislamiento. En estas circunstancias, las mujeres que sufren violencia de género permanecieron encerradas junto a su agresor, una situación de alto riesgo caracterizada por el incremento de factores de estrés en la que se pueden desencadenar con más facilidad actos violentos o de abuso (Sosa, 2020).

El confinamiento supuso una convivencia forzosa y continua con otras personas, que puede estar modulada por muchas variables psicosociales (condiciones materiales y de servicios de la vivienda, relaciones previas entre los convivientes, habilidades para la negociación y la solución de problemas, etcétera). Sus efectos en el ejercicio de la violencia por parte de los maltratadores durante y después del periodo de confinamiento, y en la salud física, mental y social de quienes la han padecido, constituyó una de las consecuencias de esta situación que es necesario afrontar (Ferrer-Pérez, 2020).

El encierro creó las condiciones ideales para el crecimiento de los elementos involucrados en la violencia de género porque el aislamiento que sufrieron las mujeres fue aún mayor, el control en el hogar aumentó, se estimuló la impunidad y se creó un contexto que facilitó el uso de cualquiera forma de violencia ante el menor estímulo (Lorente, 2020). Según Carrasco y Martínez-Reina (2020) el aislamiento social obligatorio colocó a las mujeres que convivían con sus agresores en un lugar de mayor riesgo.

Los circunstancias y factores que aumentaron de manera dramática el riesgo de violencia de la mujer durante el confinamiento fueron:

1. Convivencia con el agresor. Las mujeres se vieron obligadas a pasar mucho tiempo aisladas con su agresor, con poca posibilidad para

Tabla 2.

Consecuencias de la pandemia por Covid-19 en mujeres víctimas de violencia de género.

\section{Consecuencias}

1. Aumento de desigualdad.

2. Sobrecarga de tareas destinada al cuidado.

3. Aislamiento o distanciamiento sociofamiliar.

4. Desempleo y precarización en el trabajo.

5. Dependencia económica.

6. Estrés económico.

7. Empeoramiento de la salud física y sexual.

8. Problemas de salud mental.

9. Dificultades en los desplazamientos y limitaciones de movilidad.

10. Dificultades de acceso a recursos de apoyo.

11.Dificultades de acceso a la justicia.

\section{Fuente}

López-Hernández y Rubio-Amores (2020); Montenegro y Montenegro (2020).

Gallo y Mañas (2020).

Bagwell-Gray y Bartholmey (2020); Borges (2020); Carrasco y MartínezReina (2020); Gallo y Mañas (2020); Izquierdo (2020); Lorente (2020); Sosa (2020); Jarnecke y Flanagan (2020); Kofman y Garfin (2020); Leslie y Wilson (2020); López-Hernández y Rubio-Amores (2020).

Bedoya et al. (2020); Leslie y Wilson (2020); Sharma y Borah (2020); Montenegro y Montenegro (2020).

Lorente (2020); Ruiz-Pérez y Pastor-Moreno (2020).

Bedoya et al. (2020); Zhang (2020); Leslie y Wilson (2020); Ruiz-Pérez y Pastor-Moreno (2020).

Ferrer-Pérez (2020): Gallo y Mañas (2020); Izquierdo (2020); Sabri et al. (2020); Sharma y Borah (2020).

Ferrer-Pérez (2020); Gallo y Mañas (2020); Izquierdo (2020); Lorente (2020); Raj et al. (2020); Sediri et al. (2020); Montenegro y Montenegro (2020).

Izquierdo (2020); Ruiz-Pérez y Pastor-Moreno (2020).

Bedoya et al. (2020); Gallo y Mañas (2020); Izquierdo (2020); Zhang (2020); Sosa (2020); Kofman y Garfin (2020); Londoño (2020); López-Hernández y Rubio-Amores (2020); Montenegro y Montenegro (2020); Moreira y Pinto da Costa (2020).

Carril (2020); Londoño (2020). 
crear o fortalecer una red de apoyo (López-Hernández y Rubio-Amores, 2020; Organización Mundial de la Salud [OMS], 2020; Kofman y Garfin, 2020).

2. Poco acceso a espacios privados; de ahí que muchas mujeres tuvieran muchas dificultades para hacer una Ilamada o buscar ayuda on line (Bedoya et al., 2020).

3. Limitaciones de movilidad física y restricciones de circulación que facilitaron el comportamiento de control de los maltratadores (Ruiz-Pérez y Pastor-Moreno, 2020) y dificultaron la autonomía para salir del domicilio (Izquierdo, 2020).

4. Distanciamiento social y disminución de actividades comunitaria (Izquierdo, 2020), que imposibilitaron que las mujeres pudieran contactar con su red de apoyo social (familiares, vecinos y amigos) (López-Hernández y Rubio-Amores, 2020; OMS, 2020).

5. Sentimientos depresivos. Las circunstancias del confinamiento dieron lugar a sentimientos de impotencia y de no escapatoria (Lorente, 2020). Las mujeres con antecedentes de violencia de género parecían tener un mayor riesgo de padecer síntomas de depresión y/o ansiedad durante el confinamiento (Raj et al., 2020; Sediri et al., 2020).

6. Estrés, sobre todo por la situación y por las posibles pérdidas económicas o laborales (OMS, 2020).

7. Aumento del consumo de alcohol. Dado que la intoxicación por alcohol es un factor precipitante de la violencia de género, el riesgo de que las personas usen la violencia podría haber crecido de manera exponencial (Cafferky et al., 2018).

8. Pocas posibilidades para cambiar su vida después de una posible ruptura por las circunstancias sociales y económicas (Lorente, 2020).

9. Dificultades para pedir ayuda. El confinamiento puso en peligro a las mujeres porque las familias volvieron al ámbito privado y colocaron a las mujeres en una situación de aislamiento y amenazante con más dificultades para pedir ayuda (Izquierdo, 2020), y para acceder a servicios públicos en donde buscar protección legal y social (López-Hernández y Rubio-Amores, 2020).

10. Dificultades para interponer una denuncia. El acceso a la justicia, fiscalías o comisarías, e incluso a los abogados, fue casi imposible (Carril, 2020).

11. Dificultades para huir de estas situaciones violentas o para acceder a los escasos servicios de protección y/o servicios esenciales que pudieran salvar sus vidas (Sosa, 2020) por estar permanentemente vigiladas por los agresores (OMS, 2020).

\section{Vulnerabilidad, Covid-19 y violencia de género}

La vulnerabilidad de las mujeres sometidas a violencia de género se agrava en situaciones como la experimentada durante la pandemia por Covid-19. Según Moreira y Pinto da Costa (2020), ésta pudo influir y exacerbar aún más los factores individuales, de relación y comunitarios y sociales que precipitan los episodios de violencia en la pareja. Además, la situación que se generó a consecuencia del estrés, la perturbación de las redes sociales y de protección, y el menor acceso a los servicios incrementó el riesgo de violencia contra la mujer. Este fenómeno sociofamiliar fue en aumento porque a medida que se implantaban las medidas de distanciamiento y se pedía a las personas que se quedaran en sus casas, aumentaba la probabilidad de mayor exposición a violencia en las mujeres con una relación de maltrato. Según Bedoya et al. (2020):

"[...] además de por un mayor estrés, el aumento de tiempo que la familia permanece en contacto y las posibles pérdidas económicas o del trabajo son factores importantes a considerar" (p. 11).

Peterman et al. (2020) plantearon tres vías principales que vinculan la pandemia con la violencia contra las mujeres:

1. La inseguridad económica y el estrés relacionado con la pobreza durante el confinamiento.

2. El aislamiento social e imposibilidad de las mujeres para escapar temporalmente de sus parejas abusivas.

3. La limitada accesibilidad a los servicios de salud.

No obstante, hay que tener en cuenta que el riesgo en este contexto podría haber estado determinado en gran medida por factores de riesgo individuales y la historia de violencia previa al confinamiento, que causaría un mayor efecto en las formas de violencia y la repetición de agresiones (Lorente, 2020).

Además, Esteve (2020), integrante de la Asociación de Mujeres Juezas de España, indicó que los factores que frenan a las mujeres que podrían estar planteándose tomar la decisión de salir de una situación de violencia son:

1. Dificultad de interponer una denuncia pese a que los juzgados sigan funcionando. 
2. Imposibilidad de asistir a una consulta médica de manera presencial.

3. Falta de oportunidades para exteriorizar la situación de violencia de género, por lo que casi siempre permanecen latentes.

Es necesario considerar que esta situación causó una menor visibilidad de situaciones violentas que ocurrían en el interior de los hogares, porque las medidas adoptadas por los gobiernos de quedarse en casa, la reducción de jornada laboral o teletrabajo, y la poca disponibilidad de los servicios de protección, de prevención y de atención ocasionaron que las situaciones de violencia fueran más difíciles de denunciar (Marques et al., 2020; Londoño, 2020). Es posible que durante la desescalada aparezcan otros factores que aumenten la situación de riesgo de la mujer. La situación de estrés, derivada de nuevos miedos, con la posible situación de pérdidas económicas y la percepción de pérdida de control por parte del agresor, podrían constituir un estado de especial vulnerabilidad y riesgo (Izquierdo, 2020).

\section{Discusión}

La realización de revisiones como la que se presenta en este artículo constituye un punto de partida para visibilizar la violencia de género y aprender, para que en futuras pandemias se atiendan específicamente los casos de la violencia contra las mujeres. En ella se ha evidenciado que aunque existe un buen número de estudios pertinentes, aún se debe recorrer un largo camino con investigaciones longitudinales que revelen datos más sólidos y definan con más claridad el impacto de la pandemia Covid-19 en las víctimas de violencia de género.

Muchos autores han advertido de la invisibilidad de la violencia de género durante el periodo de confinamiento. Varios informes sugieren el aumento de la incidencia de la violencia en general y no sólo en número sino también en gravedad (Sharma y Borah, 2020), e indican que continúa siendo un problema de salud pública y que, en situaciones de emergencia como la actual, la salud de las mujeres está gravemente amenazada. No obstante, hay que destacar que pese a la agudización e incuestionable incremento de la violencia de género, los métodos de registros habituales no han sido capaces de detectarla, urgiendo la implementación de nuevos procedimientos que faciliten una visión real de esta realidad y aumenten la detección de casos.

Existen ya algunos datos que podrían indicar que la pandemia por Covid-19 ha tenido un impacto negativo en la violencia de género. En general, el confinamiento ha incidido en el aumento del aislamiento sociofamiliar, del desempleo o inestabilidad laboral, de la pobreza y del estrés, e inseguridad económica, etcétera, todos factores estresantes, grandes potenciadores de la violencia de género. Además, esta circunstancia ha tenido lugar en un contexto en el que la prioridad fue la lucha contra la pandemia, dedicando poca o nula atención a las víctimas de violencia de género.

Algunas influencias específicas del confinamiento en la violencia de género puestas de manifiesto en la literatura revisada han estado relacionadas con las dificultades para pedir ayuda, para huir o para poder denunciar la situación de violencia de género. A nivel individual se ha constatado un aumento de sintomatología depresiva y estrés. Asimismo se ha evidenciado una situación paradójica durante el confinamiento, porque a medida que aumentaba la convivencia con el agresor disminuían las relaciones de apoyo significativas y las actividades sociales y comunitarias de las mujeres víctimas de violencia de género.

La vulnerabilidad de las mujeres sometidas a violencia de género durante la pandemia por Covid-19 está determinada sobre todo por la situación de aislamiento y la reducción de las opciones de apoyo psicosocial. Las medidas de aislamiento social impuestas para minimizar el riesgo de contagio, junto con el debilitamiento de la economía y el aumento de la inestabilidad laboral, han colocado en una situación de mayor riesgo a las familias donde la violencia estaba presente en sus vidas.

En estos momentos la necesidad de visibilizar la violencia de género es una prioridad. La implicación de entidades públicas y privadas (de salud, jurídicas, educativas, etcétera) en esta tarea es fundamental; para ello es imprescindible seguir potenciando todo tipo de medidas de protección y reforzar las redes de apoyo tanto formales (familia, amigos, vecinos, etcétera) como informales tan importantes en la prevención de la violencia de género.

Ante la realidad actual en que coexisten la violencia de género y la pandemia, no queda más remedio que continuar haciendo una profunda reflexión, lo que implica:

1. Reforzar y enfatizar los recursos personales y sociales que permitan a las mujeres que están viviendo esta situación junto a su agresor lograr un empoderamiento para salvaguardar su salud física y mental.

2. Intensificar las campañas de promoción y concienciación que integren estrategias de prevención de la violencia de género. 
3. Dar apoyo a las mujeres con medidas psicosociales eficaces que actúen contra la violencia de género.

4. Analizar la efectividad real de las estrategias que se han propuesto durante el proceso (campañas informativas, mecanismos telemáticos para solicitar ayuda, incentivar conductas de ayuda del entorno, etcétera), e identificar las estrategias a usar en la actualidad y en el futuro para mitigar el riesgo de violencia de género durante las crisis sanitarias.

5. Hacer estudios científicos rigurosos para identificar los mecanismos específicos que relacionan la pandemia con el incremento de riesgo de violencia de género.

Incidir en que la violencia de género es un problema de todos y todas que sufren las mujeres. Es necesario que todos y todas las personas se posicionen en su contra, implicando al hombre en igualdad y aislando al maltratador para que comprenda que en este nuevo modelo de sociedad no es aceptable su comportamiento (Martínez, 2017).

Respecto a las limitaciones de este estudio, aunque se ha intentado hacer un búsqueda metódica y ordenada para identificar los estudios más relevantes, se han encontrado algunas restricciones en el acceso a algunos estudios publicados por distintas razones: artículos en bases de datos distintas a las utilizadas, artículos no indexados, artículos sin acceso abierto, artículos en otros idiomas no incluidos en los criterios de inclusión, etcétera. Por otro lado, el sesgo de selección puede influir de modo significativo en la revisión hecha porque se ha intentado buscar una relación entre el agravamiento de la violencia de género durante el confinamiento por Covid-19, y es posible que se hayan seleccionados los informes más accesibles y se hallan ignorado otros que podrían aportar información importante por no estar en las bases de datos consultadas.

En este sentido, sería necesario efectuar estudios más sólidos y sistemáticos en el futuro para comprender y evidenciar el impacto del Covid-19 en la violencia de género. No obstante, este trabajo supone una reflexión inicial del tema que puede ayudar a establecer estrategias de intervención adecuadas que garanticen la seguridad de las víctimas, y combatan los efectos adversos del Covid-19 en la violencia de género.

Para finalizar, la violencia contra las mujeres requiere una lucha fundamental a largo plazo y estrategias prácticas de intervención. Estos cambios deben emanar de las propias sociedades y no imponerse para cambiar de manera eficaz las mentalidades. Esto comienza con la educación dentro del núcleo familiar y requiere la colaboración entre la sociedad civil y el gobierno para promover cambios reales duraderos (Sediri et al., 2020).

\section{Conclusión}

La pandemia por Covid-19 ha impactado de modo negativo en la violencia de género, en un escenario donde las mujeres víctimas se han visto obligadas a estar confinadas con su agresor y convertirse en victimas ocultas. Por lo general la víctima se ha encontrado aislada junto a su maltratador y sin apoyo de sus redes sociofamiliares o de entidades y de servicios públicos de atención y protección. Las circunstancias sociales y económicas causadas por la pandemia de Covid-19 han creado las circunstancias perfectas para que aparezcan conductas de maltrato hacia las mujeres y generen un aumento de la vulnerabilidad. Las consecuencias se han reflejado en casi todas las áreas de la vida de las mujeres víctimas de violencia de género, potenciando los factores de riesgo de violencia de género, a nivel individual y social, al aumentar el aislamiento y las barreras que dificultan la solicitud de ayuda y la denuncia, y apareciendo con más frecuencia problemas psicoafectivos como la depresión. Todavía son necesarios datos más consistentes para definir con claridad el impacto de la pandemia por Covid-19 en las víctimas de violencia de género. No obstante, ya hay resultados importantes a tener en cuenta cuando se implementen intervenciones para prevenir la violencia de género y proteger a las víctimas de violencia de género en el contexto de una pandemia mundial.

\section{Referencias}

Referencias marcadas con asterisco indican estudios incluidos en el análisis.

Alfonso, I. (1995). Técnicas de investigación bibliográfica. Caracas: Contextos Ediciones.

Anastario, M., Shehab, N., \& Lawry, L. (2009). Increased genderbased violence among women internally displaced in Mississippi 2 years post-hurricane Katrina. Disaster Medicine and Public Health Preparedness, 3(1), 18-26. https://doi.org/10.1097/DMP.0b013e3181979c32

Bagwell-Gray, M. E., \& Bartholmey, E. (2020). Safety and Services for Survivors of Intimate Partner Violence: A ResearcherPractitioner Dialogue on the Impact of Covid-19. Psychological Trauma: Theory, Research, Practice, and Policy, 12(S1), S205-S207. http://dx.doi.org/10.1037/ $\underline{\operatorname{tra} 0000869}$

Bedoya, M. D. P., Bedoya, B. O., \& Baquero, O. X. (2020). Covid-19 y la violencia contra la mujer. Recimundo, 4(4), 242-249. https://doi.org/10.26820/recimundo/4.(4). 
octubre.2020.242-249

Bettinger-Lopez, C., \& Bro, A. (2020). A Double Pandemic: Domestic Violence in the Age of Covid-19. Domestic Violence Report, 25(5), 85-100.

Borges, R. (2020). Víctima de violencia de género y confinamiento por coronavirus: Un factor más de riesgo. Recuperado el 2 de noviembre de 2020 de https://idibe.org/tribuna/ victima-violencia-genero-confinamiento-coronavirusfactor-mas-riesgo/

Bowlus, A. J., \& Seitz, S. (2006). Domestic violence, employment, and divorce. International Economic Review, 47(4), 1113-1149. $\underline{2354.2006 .00408 . x}$

Cafferky, B. M., Mendez, M., Anderson, J. R., \& Stith, S. M. (2018). Uso de sustancias y violencia de pareja íntima: Una revisión metaanalítica. Psicología de la violencia, 8, 110131. https://doi.org/10.1037/vio0000074

Campbell, A. M. (2020). An increasing risk of family violence during the Covid-19 pandemic: Strengthening community collaborations to save lives. Forensic Science International: Reports, 2, 1-3. doi: https://doi.org/10.1016/j. fsir.2020.100089

Carrasco, L., \& Martínez-Reina, M. J. (2020). Riesgos inminentes, cuerpos descorporizados, silencios que gritan, luchas colectivas o muerte. Efectos de la pandemia Covid-19 en la configuración de las violencias contra las mujeres. Red Sociales, Revista del Departamento de Ciencias Sociales, 7(2), 46-57. http://www.redsocialesunlu.net/? $p=1498$

Carril, M. P. (2020). La violencia de género en el contexto de pandemia. Una obligada reflexión sobre la eficacia de los mecanismos de seguimiento a víctimas de violencia. Revista difusiones, 18, 47-58. https://revistadifusiones. net/index.php/difusiones/article/view/222/288

Chan, K. L., \& Zhang, Y. (2011). Female victimization and intimate partner violence after the May 12, 2008, Sichuan earthquake. Violence and Victims, 26(3), 364376. https:// doi.org/10.1891/0886-6708.26.3.364

Consejo General del Poder Judicial (CGPJ) (2020). Violencia de género. Datos y estadísticas. Recuperado el 2 de noviembre de 2020 de https://www.epdata.es/datos/ violencia-genero-estadisticas-ultima-victima/109/ espana/106

Coohey, C. (2007). The relationship between mothers' social networks and severe domestic violence: A test of the social isolation hypothesis. Violence and Victims, 22(4), 503-512. https://doi.org/10.1891/08866700778155400

Corsi, J. ( 1999). Violencia familiar: Una mirada interdisciplinaria sobre un grave problema social. Buenos Aires: Paidós.

Delegación de Gobierno contra la Violencia de Género (Ministerio de Igualdad) (2020). Violencia de género. Datos y estadísticas. Recuperado el 2 de noviembre de 2020 de https://www.epdata.es/datos/violencia-generoestadisticas-ultima-victima/109/espana/106

Esteve, L. (2020). Por qué descienden los asuntos en juzgados de violencia de género mientras aumentan las consultas al 016. Recuperado el 1 de abril de 2020 de https://www. elsaltodiario.com/coronavirus/violencia-genero-desciendedatos-policia-juzgados-aumentan-consultas-016

Ferrer-Perez, V. A. (2020). Coping with the Covid-19 pandemic and its consequences from the vantage point of feminist social psychology. International Journal of Social Psychology, 35(3), 639-646. https://doi.org/10.1080/02 134748.2020 .1783839

Fondo de Población de las Naciones Unidas (UNFPA) (2020). Covid-19: Un enfoque de género. Proteger la salud y los derechos sexuales y reproductivos y promover la igualdad de género. https://www.unfpa.org/sites/default/files/ resource-pdf/COVID-19 A Gender Lens_Guidance Note.docx_en-US es-MX.pdf

Gallo, M. T., \& Mañas, E. (2020). Territorios vulnerables a la violencia de género en tiempos de confinamiento. Madrid: Instituto Universitario de Análisis Económico y Social, Serie documentos de trabajo. http://www.iaes.es/ uploads/2/0/8/6/20860996/dt_05_20.pdf

Gearhart, S., Perez-Patron, M., Hammond, T. A., Goldberg, D. W., Klein, A., \& Horney, J. A. (2018). The impact of natural disasters on domestic violence: An analysis of reports of simple assault in Florida (1999-2007). Violence and Gender, 5(2), 87-92. https://doi.org/10.1089/vio.2017.0077

Heise, L., Pitanguy J., \& Germain, A. (1994). Violencia contra la mujer: La carga oculta sobre la salud. Programa mujer, salud y desarrollo. Washington, D. C.: Oficina Sanitaria Panamericana, Oficina Regional de la Organización Mundial de la Salud.

Izquierdo, R. (2020). Violencia de género en tiempo de Covid-19. Cadernos de Atención Primaria, 26(2), 26-27. https:// revista.agamfec.com/wp-content/uploads/2020/07/ Agamfec-26 2-Violencia-de-g\%C3\%A9nero-en-tiemposdel-covid-19.pdf

Jarnecke, A. M., \& Flanagan, J. C. (2020). Staying safe during Covid-19: How a pandemic can escalate risk for intimate partner violence and what can be done to provide individuals with resources and support. Psychological Trauma: Theory, Research, Practice and Policy, 12(S1), S202-S204. http://dx.doi.org/10.1037/tra0000688

Kofman, Y. B., \& Garfin, D. R. (2020). Home Is Not Always a Haven: The Domestic Violence Crisis Amid the Covid-19 Pandemic. Psychological Trauma: Theory, Research, Practice, and Policy, 12(S1), S199-S201. http://dx.doi. org/10.1037/tra0000866

Leslie, E., \& Wilson, R. (2020). Sheltering in place and domestic violence: Evidence from calls for service during Covid-19. Journal of Public Economics, 189, 1-7. https://doi. org/10.1016/j.jpubeco.2020.104241

Ley Orgánica $1 / 2004$ de 28 de diciembre, de medidas de protección integral contra la violencia de género. Boletín Oficial del Estado, 313, de 3 de diciembre de 2004, 4216642197. https://www.boe.es/boe/dias/2004/12/29/ pdfs/A42166-42197.pdf

Londoño, N. (2020). Expresiones de la violencia basada en género, en el marco del confinamiento por Covid-19. Nova, 18(35), 107-113. https://doi.org/10.22490/24629448.4194

López-Hernández, E., \& Rubio-Amores, D. (2020). Reflexiones sobre la violencia intrafamiliar y violencia de género durante emergencia por Covid-19. CienciAmérica, 9(2), 312-321. http://dx.doi.org/10.33210/ca.v9i2.319

Lorente, M. (2020). Gender-based violence during the pandemic and lockdown. Spanish Journal of Legal Medicine, 46(3), 
139-145. https://doi.org/10.1016/j.reml.2020.05.005

Marques, E. S., de Moraes, C. L., Hasselmann, M. L., Deslandes, S. F., \& Reichenheim, M. E. (2020). Violencia contra mujeres, niños y adolescentes en tiempos de la pandemia de Covid-19: Panorama, motivaciones y formas de afrontamiento. Cadernos de Saúde Pública, 36(4), 1-6. https://doi.org/10.1590/0102-311x00074420

Martínez, E. (2017). La igualdad y la violencia de género: elementos para la reflexión en España y en Europa. En J. Hurtado Pozo (dir.), Género y Derecho Penal (169-170). Lima: Instituto Pacífico.

Ministerio de Sanidad, Servicios Sociales e Igualdad (MSSSI) (2020). Violencia de género. Datos y estadísticas. Recuperado el 2 de noviembre de 2020 de https://www. epdata.es/datos/violencia-genero-estadisticas-ultimavictima/109/espana/106

Montenegro, E., \& Montenegro, M. (2020). Re-pensando el campo de intervención del trabajo social junto a las víctimas de violencia de género, durante la pandemia de Covid-19. Revista Difusiones, 18(18), 59-67. https:// revistadifusiones.net/index.php/difusiones/article/ view/223/289

Moreira, D. N., \& Pinto da Costa, M. (2020). The impact of the Covid-19 pandemic in the precipitation of intimate partner violence. International Journal of Law and Psychiatry, 71, 101606. https://doi.org/10.1016/j.ijlp.2020.101606

Nogueiras, B. (2005). La violencia en la pareja. En C. RuizJarabo y P. Blanco (dirs.). La violencia contra las mujeres. Prevención y detección (pp. 39-56). Madrid: Díaz de Santos.

Organización Mundial de la Salud (OMS) (2020). Covid-19 and violence against women What the health sector/system can do. Recuperado el 25 de noviembre de 2020 de https://www.who.int/reproductivehealth/publications/ emergencies/COVID-19-VAW-full-text.pdf

Osborne, R. (2009). Apuntes sobre violencia de género. Barcelona: Bellaterra.

Otero-García, L., Briones-Vozmediano, E., Vives-Cases, C., García-Quinto, M., Sanz-Barbero, B., \& Goicolea, I. (2018). A qualitative study on primary health care responses to intimate partner violence during the economic crisis in Spain. The European Journal of Public Health, 28(6), 10001005. https://doi.org/10.1093/eurpub/cky095

Parkinson, D. (2019). Investigating the increase in domestic violence post disaster: An australian case study. Journal of Interpersonal Violence, 34(11), 2333-2362. https://doi. org/10.1177/0886260517696876

Peprah, J. A., \& Koomson, I. (2017). Impulsores económicos de la violencia doméstica entre las mujeres: Un estudio de caso sobre la violencia y la sociedad en Ghana: Avances en la investigación y la práctica (222-240). IGI global.

Peterman, A., Potts, A., O'Donnell, M., Thompson, K., Shah, N., Oertelt-Prigione, S., \& Van Gelder, N. (2020). Pandemics and violence against women and children. Center for Global Development, Working Paper, 528. https://www. cgdev.org/sites/default/files/pandemics-and-vawg-april2. pdf

Raj, A., Johns, N. E., Barker, K. M. \& Silverman, J. G. (2020). Time from Covid-19 shut down, gender-based violence exposure, and mental health outcomes among a state representative sample of California residents. EClinicalMedicine, 26, 100520. https://doi.org/10.1016/j. eclinm.2020.100520

Real Decreto-Ley 12/2020, de 31 de marzo, de medidas urgentes en materia de protección y asistencia a las víctimas de violencia de género. Boletín Oficial del Estado, 91, de 1 de abril de 2020, 27973-27980. https://www.boe.es/eli/es/ $\mathrm{rdl} / 2020 / 03 / 31 / 12$

Reingle Gonzalez, J. M., Molsberry, R., Maskaly, J., \& Jetelina, K. K. (2020). Las tendencias en violencia familiar no están asociadas causalmente con las órdenes de estancia en el hogar Covid-19: Un comentario sobre Piquero et al. American Journal of Criminal Justice, 45, 1100-1110. https://doi.org/10.1007/s12103-020-09574-w

Ruiz-Pérez, I., \& Pastor-Moreno, G. (2020). Medidas de contención de la violencia de género durante la pandemia de Covid-19. Gaceta Sanitaria, 1-6. https://doi. org/10.1016/i.gaceta.2020.04.005

Sabri, B., Hartley, M., Saha, J., Murray, S., Glass, N., \& Campbell, J. C. (2020). Effect of Covid-19 pandemic on women's health and safety: A study of immigrant survivors of intimate partner violence. Health Care for Women International, 41(11-12), 1294-1312. https://doi.org/10.1080/073993 32.2020.1833012

Sakurai, K., Nishigori, H., Nishigori, T., Mizuno, S., Obara, T., Iwama, N., ..., Yaegashi, N. (2017). Incidence of Domestic Violence Against Pregnant Females After the Great East Japan Earthquake in Miyagi Prefecture: The Japan Environment and Children's Study. Disaster Medicine and Public Health Preparedness, 11(2), 216-226. https://doi. org/10.1017/dmp.2016.109

Sánchez-Meca, J. (2010). Como realizar una revisión sistemática y un metaanálisis. Aula Abierta, 38, 2, 53-64.

Sediri, S., Zgueb, Y., Ouanes, S. Ouali, U., Bourgou, S., Jomli, R., \& Nacef, F. (2020). Salud mental de las mujeres: Impacto agudo de la pandemia Covid-19 en la violencia doméstica. Archives of Women's Mental Health, 23, 749-756. https:// doi.org/10.1007/s00737-020-01082-4

Sharma, A., \& Borah, S. B. (2020). Covid-19 and Domestic Violence: An Indirect Path to Social and Economic Crisis. Journal of Family Violence. https://doi.org/10.1007/ s10896-020-00188-8

Sosa, S. A. (2020). Empoderamiento y violencia de género en contexto de cuarentena. Revista Digital Prospectivas en Psicología, 4(2), 16-28. https://www.kennedy.edu. ar/wp-content/uploads/2020/08/05_04_02_2.EMPODERAMIENTO-y-VIOLENCIA-DE-GENERO-ENCONTEXTO-DE-CUARENTENA.pdf

Taub, A. (2020). A new Covid-19 crisis: Domestic abuse rises worldwide. The New York Times Company, 9. Recuperado el20 denoviembre de 2020 de https://www.chicagotribune. $\mathrm{com} /$ coronavirus/sns-nyt-coronavirus-domestic-abuserises-worldide-20200407-6kd46ga4hrfizoxmhz4dmiigjastory.html

Urrutia, G., \& Bonfill, X. (2010). Declaración Prisma: Una propuesta para mejorar la publicación de revisiones sistemáticas y metaanálisis. Medicina Clínica, 135(11) 507-511. https://doi.org/10.1016/j.medcli.2010.01.015 


\section{Meta-Análisis del Artículo}




\section{Dimensión Cuantitativa}

\section{Perfil de Evaluación entre pares}
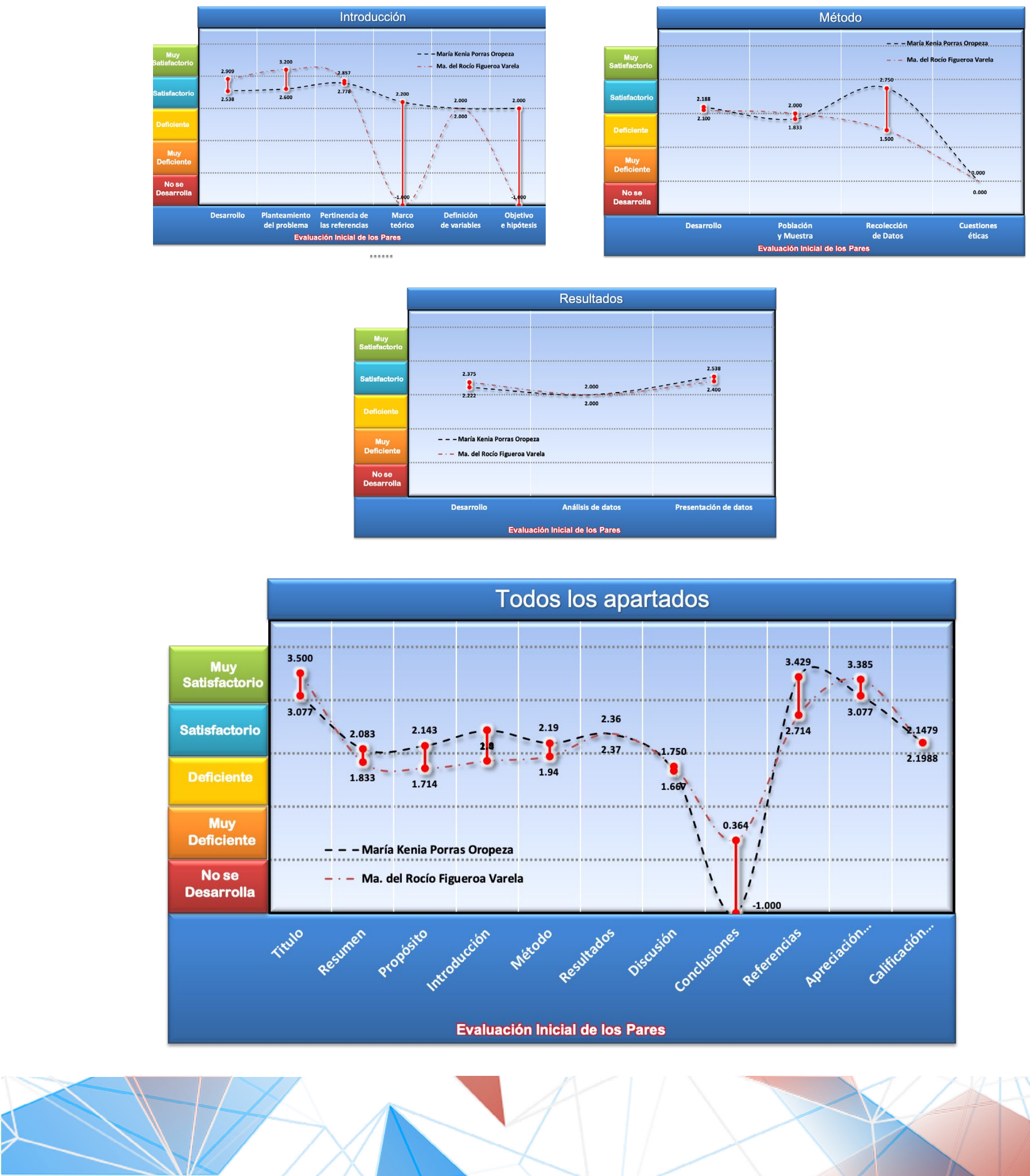


\section{Índice de Concordancia}

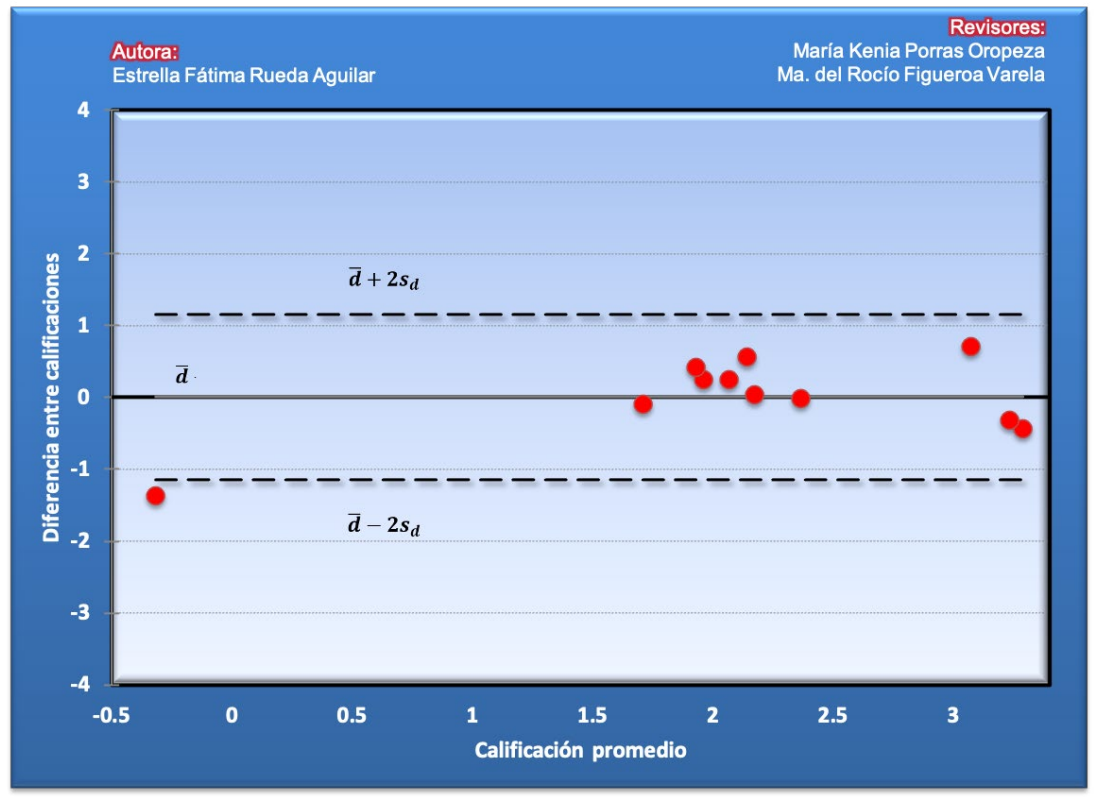

Índice de Acuerdo

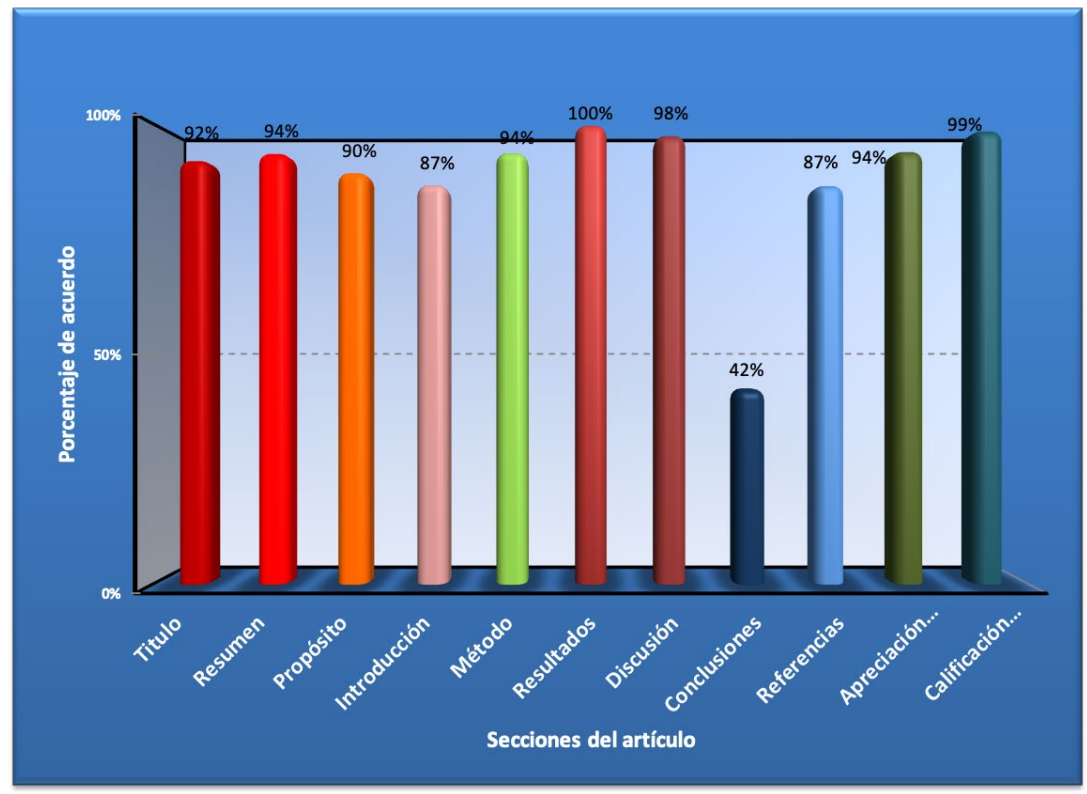




\begin{tabular}{|l|}
\hline \multicolumn{1}{|c|}{ Revisor 1 } \\
\hline \multicolumn{1}{|c|}{ María Kenia Porras Oropeza } \\
\hline Título/A \\
$\begin{array}{l}\text { El título puede ser más concreto para que corresponda } \\
\text { con el contenido presentado, puede indicar el país, el gru- } \\
\text { po de edad de la población estudiada y la razón del con- } \\
\text { finamiento. }\end{array}$ \\
\hline
\end{tabular}

Revisor 2

Ma. del Rocío Figueroa Varela

\section{Resumen}

Hay una introducción al inicio del resumen que resta espacio a los elementos que debe contener y faltan (metodología, resultados y conclusiones).

Rehacer el resumen para indicar con claridad el propósito del documento, su método para desarrollarlo, los resultados más relevantes y las conclusiones pertinentes.

\section{Próposito del Estudio}

Aunque la redacción es clara, falta especificidad al objetivo y no se retoman las preguntas derivadas de él en los resultados, en la discusión ni en las conclusiones.

Dada la profusa publicación de escritos sobre el tema, la originalidad de los aportes está disminuida. Se sugiere revisar con detenimiento el método declarado, pues si bien el problema es actual y vigente, las preguntas de investigación no son contestadas a través del método enunciado. Dado que el propósito fue "la sistematización objetiva y metódica de los estudios publicados con el objetivo de concretar el estado de arte de la cuestión" no se revisa con claridad en la discusión este estado de arte que se enunció en el método.

\section{Introducción}

El problema de investigación puede plantearse apoyándose en alguno de los marcos teóricos de los estudios de género que ya existen. Definir las nociones conceptuales e incluirlas en las preguntas para orientar la presentación y análisis de los resultados.

Es confuso que se presentan unas preguntas que parecería deberían de guiar su estudio, sin embargo no se incluyen términos en la revisión que están en relación con las preguntas planteadas, en específico las de violencia familiar o de cifras ocultas de la violencia. Por lo tanto precisar un marco de referencia teórico ayudaría a revisar desde dónde se está viendo el fenómeno para ser explicado. Así también mejorar la congruencia entre las preguntas, el propósito y el método ayudarían a identificar aspectos relevantes para investigar ya sea la prevalencia o la problemática asociada a la violencia de género 


\begin{tabular}{|c|c|}
\hline Revisor 1 & Revisor 2 \\
\hline \multicolumn{2}{|c|}{ Método } \\
\hline $\begin{array}{l}\text { Se recomienda organizar por secciones su contenido. Se- } \\
\text { ñalar de forma explícita en el procedimiento el periodo } \\
\text { de las publicaciones revisadas. Agregar la descripción de } \\
\text { cada uno de los criterios de inclusión ya que son gene- } \\
\text { rales. Incluir la justificación del tamaño de la muestra a } \\
\text { la luz de estado del arte del tema investigado. Incluir un } \\
\text { apartado sobre estrategias de análisis para saber cómo } \\
\text { fue procesada y organizada la información de las publi- } \\
\text { caciones para llegar a la muestra final. }\end{array}$ & $\begin{array}{l}\text { A pesar de que cita a dos documentos en donde asegu- } \\
\text { ra se basa su método, al revisarse se observa que no se } \\
\text { siguen las mismas recomendaciones para hacer las re- } \\
\text { visiones sistemática, por lo tanto no se puede alcanzar } \\
\text { el objetivo del estudio ni se llega a contestar las pregun- } \\
\text { tas de investigación. Se sugiere identificar con claridad } \\
\text { el método elegido, su forma de análisis de información } \\
\text { y que se siga también los pasos recomendados por los } \\
\text { mismos autores que han sido citados. Además es im- } \\
\text { portante identificar el periodo de la búsqueda de infor- } \\
\text { mación, puesto que sin hacer una búsqueda detallada se } \\
\text { localizaron los documentos que se enlistan al finalizar, } \\
\text { que no se integraron en este análisis, lo cual indica que } \\
\text { se han producido muchos artículos sobre el tema y para } \\
\text { el caso de esta revisión sistemática es mejor identificar } \\
\text { un periodo especifico de los documentos que se inte- } \\
\text { gren. The hidden disaster of COVID-19: Intimate part- } \\
\text { ner violence. Buttell F, Ferreira RJ.Psychol Trauma. 2020 } \\
\text { Aug;12(S1):S197-S198. doi: 10.1037/tra0000646. Trying } \\
\text { Times and Trying Out Solutions: Intimate Partner Vio- } \\
\text { lence Screening and Support for Women Veterans Du- } \\
\text { ring COVID-19. Rossi FS, Shankar M, Buckholdt K, } \\
\text { Bailey Y, Israni ST, Iverson KM.J Gen Intern Med. 2020 } \\
\text { Sep;35(9):2728-2731. doi: 10.1007/s11606-020-05990- } \\
\text { 0. Safety and services for survivors of intimate partner } \\
\text { violence: A researcher-practitioner dialogue on the } \\
\text { impact of COVID-19. Bagwell-Gray ME, Bartholmey } \\
\text { E.Psychol Trauma. 2020 Aug;12(S1):S205-S207. doi: } \\
\text { 10.1037/tra0000869. The impact of the Covid-19 pan- } \\
\text { demic in the precipitation of intimate partner violence. } \\
\text { Moreira DN, Pinto da Costa M.Int J Law Psychiatry. } \\
\text { 2020 Jul-Aug;71:101606. doi: 10.1016/jijlp.2020.101606. } \\
\text {. COVID-19, Lockdown, and Intimate Partner Violen- } \\
\text { ce: Some Data from an Italian Service and Suggestions } \\
\text { for Future Approaches. Barbara G, Facchin F, Mic- } \\
\text { ci L, Rendiniello M, Giulini P, Cattaneo C, Vercellini } \\
\text { P, Kustermann A. J Womens Health (Larchmt). 2020 } \\
\text { Oct;29(10):1239-1242. doi: 10.1089/jwh.2020.8590. }\end{array}$ \\
\hline
\end{tabular}




\begin{tabular}{|l|}
\hline Revisor 1 \\
\hline
\end{tabular}

No hay congruencia entre lo que se reporta como resultado y los documentos analizados dado que se encontraron 25 documentos citados en los resultados, pero solo 18 cumplen con el criterio de inclusión del año indicado en el método; solo un documento es un estudio en donde se basan en datos empíricos, tres de los documentos citados no se pueden considerar publicaciones académicas, los demás son reflexiones, revisiones de literatura y recomendaciones de organizaciones o instituciones sobre la posibilidad de un incremento de la violencia de género que se ha revisado en circunstancias similares. La mayoría de los documentos considera los mismos elementos por lo tanto las categorías para el análisis son muy genéricas. No se considera alcanzar, con los resultados mostrados, identificar el estado del arte que se pretendía realizar con la revisión, sobre todo porque no se profundiza en el análisis de los documentos revisados. No se identifica las lagunas ni sesgos de información, o en dónde o por quienes se está revisando el fenómeno, esto podría contribuir a mejorar el artículo. Por otro lado se presenta un gráfico y una tabla que tiene información repetida, por lo tanto se sugiere solo utilizar uno de estos elementos.

\section{Discusión}

Retomar los resultados del estudio para responder al objetivo y preguntas planteadas inicialmente y contrastarlo con lo que han señalado otrxs autorxs. Incluir y argumentar las respuestas y reflexiones surgidas del trabajo investigativo. Incluir las limitaciones metodológicas y éticas del estudio al seguir en confinamiento.

No se desarrolla una discusión como tal, solo se presentan algunas ideas a modo de conclusiones y se finaliza con algunas recomendaciones. Se sugiere revisar con detalle esta sección del escrito, sobre todo para indicar líneas de investigación o intervención que pudieran derivarse, sustentando los argumentos en la literatura revisada. También se sugiere evitar la repetición de ideas, pues en todo el documento se puede revisar que hay párrafos de contenido similar que podrían sintetizarse.

Conclusiones

Incluir el apartado de conclusiones al manuscrito.

En específico no tiene una sección de conclusiones, por lo tanto se sugiere desarrollarla en forma separada a la discusión. 
Revisión de la literatura | Reflexiones sobre la violencia de género durante el confinamiento... $\mid$ Rueda Aguilar

\begin{tabular}{|c|c|}
\hline Revisor 1 & Revisor 2 \\
\hline \multicolumn{2}{|c|}{ Referencias } \\
\hline $\begin{array}{l}\text { Las referencias son actualizadas }(76 \%) \text { sin embargo hay } \\
\text { que revisar y corregir algunas citas en el texto y en la lista } \\
\text { de referencias porque no están en el formato de la APA. }\end{array}$ & $\begin{array}{l}\text { Se sugiere indicar cuáles fueron los documentos integra- } \\
\text { dos en la revisión y cuáles fueron de sustento para la in- } \\
\text { troducción. Así también es necesario revisar con cuidado } \\
\text { el Manual de Estilo APA porque existen múltiples errores } \\
\text { tanto en las citas como en las referencias. }\end{array}$ \\
\hline
\end{tabular}

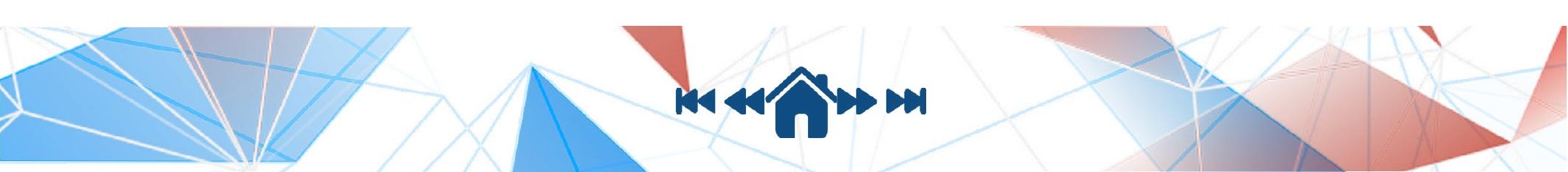


